\title{
Model-based extraction of femoral medulla ducts from radiographic images
}

\author{
Franco Bartolini ${ }^{\mathrm{a}, \uparrow}$, Monica Carfagni ${ }^{\mathrm{b}}$, Lapo Governi ${ }^{\mathrm{b}, *}$ \\ a'Dipartimento di Elettronica e Telecomunicazioni, Università di Firenze, Via Santa Marta 3, Firenze 50139, Italy

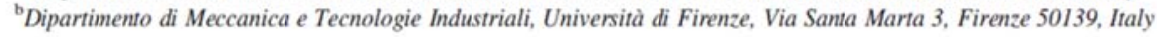 \\ Received 25 June 2002; received in revised form 3 June 2003; accepted 12 June 2003
}

\begin{abstract}
The planning of the hip prosthesis surgical operation is usually performed manually by the surgeon, who 'draws' on a patient's X-ray image the outline of the prosthetic stem in order to choose the one most suitable for the case at hand. In an attempt to give some repeatability and objectivity to the planning phase, a procedure has been devised for hip prosthesis' stem selection based on the extraction of the femoral relevant outlines.

This work presents a computer aided method aimed at automatically extracting the medulla duct outlines from a human femur radiographic image. The outlines are retrieved by referring to a suitable geometric model of the generic femoral cross-section; the projection function obtained by simulating the radiographic acquisition of such a model is fitted on the grey-level functions corresponding to the rows of the actual digitised radiographic image by means of a least squares algorithm. The resulting outlines are used in a software tool performing the hip prosthesis pre-operational planning.
\end{abstract}

(C) 2003 Published by Elsevier B.V.

Keywords: Model-based image processing; Medical images processing; Surgery aiding tools 